Technological University Dublin

DÜBLIN

ARROW@TU Dublin

Articles

School of Mathematics

1985-01-01

\title{
On Endomorphism Algebras of Mixed Modules
}

Brendan Goldsmith

Technological University Dublin, brendan.goldsmith@tudublin.ie

B. Franzen

Follow this and additional works at: https://arrow.tudublin.ie/scschmatart

Part of the Mathematics Commons

\section{Recommended Citation}

Goldsmith, B. \& Franzen, B. (1985). On endomorphism algebras of mixed modules. Journal London

Mathematical Society, vol. 2, no. 31, pg. 468-472. doi:10.1112/jlms/s2-31.3.468

This Article is brought to you for free and open access by the School of Mathematics at ARROW@TU Dublin. It has been accepted for inclusion in Articles by an authorized administrator of ARROW@TU Dublin. For more information, please contact arrow.admin@tudublin.ie, aisling.coyne@tudublin.ie,gerard.connolly@tudublin.ie. 
ON ENDOMORPHISM ALGEBRAS OF MIXED MODULES

B. FRANZEN AND B. GOLDSMITH 


\title{
ON ENDOMORPHISM ALGEBRAS OF MIXED MODULES
}

\author{
B. FRANZEN AND B. GOLDSMITH
}

\section{Introduction}

In a remarkable paper [1] some twenty years ago, Corner showed that every countable reduced torsion-free ring is the endomorphism ring of a countable reduced torsion-free abelian group. This has been the starting point for many investigations of the so-called realization problem, which may be stated as follows.

Given an algebra $A$ over a commutative ring $R$, when will $A$ be the endomorphism algebra of an $R$-module $G$ which belongs to some suitably restricted class $\mathscr{C}$. Complete characterizations of such algebras $A$ have been obtained in the case where $R$ is a complete discrete valuation ring and $\mathscr{C}$ is the class of torsion-free reduced $R$-modules [11] and also in the case where $R=\mathbb{Z}$ and $\mathscr{C}$ is the class of separable $p$-groups [10; 9, Section 109]. Such characterizations are, inevitably, much too complicated to lend themselves readily to applications. Consequently Corner [2] tackled the realization problem for primary abelian groups from a different angle. He showed that a suitably large class of rings $A$ could not be realized as full endomorphism rings, but rather that the full endomorphism algebra would be the split extension of the given ring $A$ by some ideal whose presence was unavoidable; in the case of primary groups this ideal is precisely the ideal of small endomorphisms [2]. This idea was subsequently extended to large primary groups in [6] and a similar result was produced in [8] for torsion-free modules over a complete discrete valuation ring.

The results in $[2,6,8]$ are all capable of translation into results on endomorphism algebras in a suitable quotient category. Thus, for example, if $\mathscr{S}$ is the category having primary abelian groups as objects, and morphisms

$$
\operatorname{Hom}_{\mathscr{S}}(G, H)=\operatorname{Hom}(G, H) / \operatorname{Hom}_{s}(G, H),
$$

where $\operatorname{Hom}_{s}(G, H)$ consists of the small homomorphisms of $G$ into $H$, then Corner's result is that if $A$ is a ring whose additive group is the completion of a free $p$-adic module of at-most countable rank, then there exists a primary group $G$ with $E_{\mathscr{S}}(G)=A$.

When dealing with mixed abelian groups (or more generally mixed $R$-modules), there is a natural category in which to work, viz. the category Walk $\left({ }_{R}\right.$ Walk). The objects of ${ }_{R}$ Walk are $R$-modules and its morphisms are given by

$$
\operatorname{Hom}_{W}(G, H)=\operatorname{Hom}(G, H) / \operatorname{Hom}_{t}(G, H),
$$

where $\operatorname{Hom}_{t}(G, H)$ consists of the $R$-homomorphisms of $G$ into $H$ with torsion image (see [12]). Recently Dugas [4] has shown that each torsion-free reduced ring $A$ is the Walk-endomorphism ring of a mixed abelian group $G$. The groups $G$ so realized are all of large infinite rank even when the ring $A$ is of comparatively small cardinality.

Received 14 December 1983; revised 28 May 1984.

1980 Mathematics Subject Classification 20K21.

$$
\text { J. London Math. Soc. (2) } 31 \text { (1985) 468-472 }
$$


The cardinalities of these groups have been significantly reduced in [3], which unifies the torsion, torsion-free and mixed cases of the realization problem.

Our approach will be to construct a (non-trivial) full embedding of the category of torsion-free reduced $R$-modules into the category ${ }_{R}$ Walk, where $R$ is a principal ideal domain. As a consequence of this full embedding we may immediately lift established results from the category of reduced torsion-free $R$-modules to the category ${ }_{R}$ Walk. A typical, but by no means exhaustive, list of such results is contained in Corollaries 2.4-2.6. We note, in particular, that many of the results in [7] can now be established immediately. It is, by now, standard to use such realization results to exhibit a wide range of pathologies and so we desist from such repetition.

We conclude this introduction by noting that all unexplained terms may be found in the standard works of Fuchs [9]; our notation is in accord with [9] except that maps are written on the right.

\section{The embedding theorem}

Throughout let $R$ be a principal ideal domain. We begin with an arbitrary reduced, separable torsion $R$-module $T$, and $T^{\prime}$ any pure extension of $T$ by $Q / R$ such that $T^{\prime}$ is also separable and reduced. Thus we have a pure-exact sequence of $R$-modules

$$
0 \longrightarrow T \longrightarrow T^{\prime} \longrightarrow Q / R \longrightarrow 0 ;
$$

these will be fixed for the rest of the section. Note that provided $T$ has no torsion-complete $p$-component $T_{p}$, such a sequence exists (see [9, Corollary 68.5]).

Now, if $X$ is an arbitrary $R$-module, then (*) yields another pure-exact sequence (see [9, Theorem 60.4]):

$$
0 \longrightarrow T \otimes X \longrightarrow T^{\prime} \otimes X \longrightarrow Q / R \otimes X \longrightarrow 0
$$

Since $Q / R \otimes X$ is canonically an epimorphic image of $Q \otimes X$ we can form the pullback $H(X)$ of $\left(*_{X}\right)$ with respect to this canonical epimorphism $\eta_{X}$. This yields the diagram

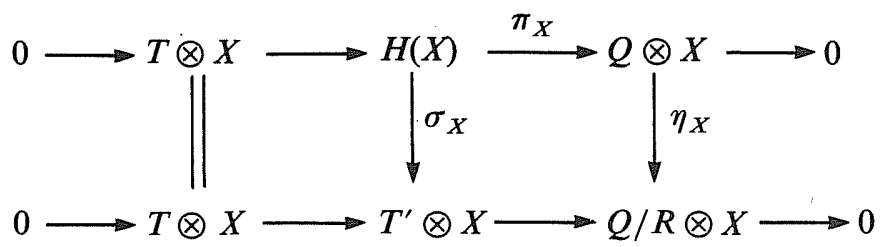

in which $\sigma_{X}$ is epic since $\eta_{X}$ is epic. Note that, by the construction of a pullback, $\operatorname{Ker} \sigma_{X}$ is mapped isomorphically onto $\operatorname{Ker} \eta_{X}$ by $\pi_{X}$. Also $\operatorname{Ker} \eta_{X}$ is canonically isomorphic to $X / t(X)$. The $R$-module $H(X)$ has the same torsion-free rank as $X$ and its torsion submodule is isomorphic to $T \otimes X$. Note that, if $X$ is torsion-free reduced, then $H(X)$ is reduced and hence non-split. If $U(M)=\bigcap_{0 \neq r \in R} r M$ denotes the first Ulm submodule of an $R$-module, then the purity of $(*)$ implies the following result.

\section{LEMMA 2.1. $\operatorname{Ker} \sigma_{X}=U(H(X))$.}

Proof. Note first that it follows from [9, Theorem 61.1] that $T_{p}^{\prime} \otimes X \cong T_{p}^{\prime} \otimes B_{p}$, where $B_{p}$ is a $p$-basic submodule of $X$. Thus $T_{p}^{\prime} \otimes X=\bigoplus_{p} T_{p}^{\prime} \otimes B_{p}$ and since $T^{\prime}$ is separable it follows readily that $U\left(T^{\prime} \otimes X\right)=0$. But $U(H(X)) \sigma_{X} \subseteq U\left(T^{\prime} \otimes X\right)=0$ and hence $U(H(X)) \subseteq \operatorname{Ker} \sigma_{X}$. 
Conversely, let $m$ be an arbitrary element of $\operatorname{Ker} \sigma_{X}$ and let $r$ be an arbitrary nonzero element of $R$. Then there is an element $y \in H(X)$ with $m-r y=z \in t(H(X))$. But then

$$
z=z \sigma_{X}=m \sigma_{X}-r y \sigma_{X}=-r y \sigma_{X} \in r\left(T^{\prime} \otimes X\right) \cap T \otimes X=r(T \otimes X)
$$

by the purity of the sequence $\left(*_{X}\right)$. Hence $m \in r H(X)$. Since $r$ is arbitrary non-zero, we have $m \in U(H(X))$ and so $\operatorname{Ker} \sigma_{X} \subseteq U(H(X))$.

We remark that the construction of $H(X)$ is functorial: every $f \in \operatorname{Hom}(X, Y)$ yields homomorphisms $Q \otimes X \rightarrow Q \otimes Y$ and $T^{\prime} \otimes X \rightarrow T^{\prime} \otimes Y$ which in turn give rise to a unique homomorphism $H(f): H(X) \rightarrow H(Y)$ by the universal property of the pullback. We denote this functor by $H$. In order to place our construction in a functorial setting let $U$ be the subfunctor of the identity defined by $U(X)=\bigcap_{0 \neq r \in R} r X$ and $U(f)=f \mid U(X)$, the restriction of $f$ to $U(X)$; let $F$ be the functor defined by $F(X)=X / t(X)$ and $F(f)=\bar{f}$, where $\bar{f}$ is the mapping induced by $f$ on the quotient.

Proposition 2.2. The functors $U H$ and $F$ are naturally equivalent.

Proof. By Lemma 2.1 $U H(X)=\operatorname{Ker} \sigma_{X}$ and since $\pi_{X}$ maps $\operatorname{Ker} \sigma_{X}$ isomorphically onto the kernel of $\eta_{X}$ the assertion follows from the observation that $\operatorname{Ker} \eta_{X} \cong X / t(X)$.

In the following let ${ }_{R}^{\mathscr{C}}$ denote the category of torsion-free reduced $R$-modules.

THEOREM 2.3. Let $R$ be a principal ideal domain, let $T$ be a separable reduced torsion $R$-module and let $T^{\prime}$ be a pure extension of $T$ by $Q / R$ such that $T^{\prime}$ is separable and reduced. Then there is a full embedding $\bar{H}:{ }_{R} \mathscr{C} \rightarrow{ }_{R}$ Walk such that

(i) $\bar{H}(X)$ is reduced, non-split and of the same torsion-free rank as $X$,

(ii) $t(\bar{H}(X)) \cong T \otimes X$,

(iii) $\bar{H}(X) / t(\bar{H}(X))$ is divisible,

(iv) $U \bar{H}(X)=X$ and $\bar{H}(X) / U \bar{H}(X)=T^{\prime} \otimes X$.

Proof. Let $\bar{H}(X)=H(X)$ for $X \in_{R} \mathscr{C}$ and $\bar{H}(f)=H(f)+\operatorname{Hom}_{t}(H(X), H(Y))$ for $f: X \rightarrow Y$. The only assertion still to be verified is that $\bar{H}$ is a full embedding. By Proposition 2.2 $U H$ is naturally equivalent to $F$ which is the identity functor on ${ }_{R}^{\mathscr{C}}$. Therefore we may identify $X$ and $U H(X)$. Consider the homomorphisms $h: \operatorname{Hom}(X, Y) \rightarrow \operatorname{Hom}(H(X), H(Y))$ and $u: \operatorname{Hom}(H(X), H(Y)) \rightarrow \operatorname{Hom}(X, Y)$ induced by $H$ and $U$ respectively. Then $h u$ is the identity on $\operatorname{Hom}(X, Y)$, thus $h$ is monic and $u$ is epic. Furthermore $\operatorname{Ker} u=\operatorname{Hom}_{t}(H(X), H(Y))$ since $g \mid U H(X)=0$ implies that $\operatorname{Im} g$ is torsion as an epimorphic image of the torsion module $H(X) / U H(X) \cong$ $T^{\prime} \otimes X$. On the other hand, if $\operatorname{Im} g$ is torsion, then $g(U H(X))=0$ because $U H(Y) \cap t(H(Y))=0$. Thus we conclude that the map $f \mapsto \bar{H}(f)$ is an isomorphism and $\bar{H}$ is a full embedding.

REMARKS. (a) An alternative way to construct the functor $H$ is the following. Let $M=H(R)$, a mixed module of torsion-free rank one. Then it is readily seen that the functors $H$ and $M \otimes$ - are naturally equivalent. 
(b) As indicated in the above proof, $E(H(X))$ is the split extension of $E(X)$ by $\operatorname{Hom}_{t}(H(X), H(X))$, that is, there are ring homomorphisms

$$
E(X) \stackrel{h}{\longrightarrow} E(H(X)) \stackrel{u}{\longrightarrow} E(X)
$$

such that $h u=\mathrm{id}_{E(X)}$ and $\operatorname{ker} u=H o m_{t}(H(X), H(X))$.

COROLlaRy 2.4. Let $R$ be a principal ideal domain. If $A$ is a countable reduced torsion-free $R$-algebra then there are $2^{\aleph_{0}}$ countable mixed $R$-modules $M_{i}$ with $M_{i} / t\left(M_{i}\right)$ divisible, $E_{W}\left(M_{i}\right) \cong A$ and $\operatorname{Hom}_{W}\left(M_{i}, M_{j}\right)=0$ for $i \neq j$.

Proof. By an unpublished extension of a well-known theorem of Corner [1] there exist countable reduced torsion-free modules with $E\left(X_{i}\right) \cong A$ and $\operatorname{Hom}\left(X_{i}, X_{j}\right)=0$ for $i \neq j$. Now Theorem 2.3 yields the assertion by choosing an appropriate torsion module $T$, for example an unbounded countable direct sum of cyclics.

In the finite rank case Corner's result gives the following.

COROLlaRY 2.5. Let $R$ be a principal ideal domain and let $A$ be a countable reduced torsion-free algebra of finite rank $n$. Then there exists a reduced mixed module $M$ of torsion-free rank $2 n$ such that $M / t(M)$ is divisible and $E_{W}(M)=A$.

COROLlaRY 2.6. If $R$ is a principal ideal domain and not a complete discrete valuation ring and $A$ is any cotorsion-free $R$-algebra, then there exists a reduced mixed $R$-module $M$ with $M / t(M)$ divisible and $E_{W}(M)=A$.

Proof. This is a consequence of [5, Corollary 5.4], which ensures the existence of a cotorsion-free $R$-module $X$ with $E(X)=A$.

REMARK. It was shown in [3] that $X$ can be chosen to be of cardinality $|A|^{\aleph_{0}}$. Thus $M$ can be made to be of cardinality $|A|^{|R|}$.

\section{References}

1. A. L. S. CoRneR, 'Every countable reduced torsion-free ring is an endomorphism ring', Proc. London Math. Soc. (3), 13 (1963), 687-710.

2. A. L. S. CORNER, 'On endomorphism rings of primary abelian groups', Quart. J. Math. Oxford, 20 (1969), 277-296.

3. A. L. S. CoRNer AND R. GöBel, 'Prescribing endomorphism algebras, a unified treatment', Proc. London Math. Soc. (3), 50 (1985), 447-479.

4. M. Dugas, 'On the existence of large mixed modules', Abelian group theory, Lecture Notes in Mathematics 1006 (eds. R. Göbel, L. Lady and A. Mader, Springer, Berlin, 1983), pp. 412-424.

5. M. DugAS AND R. GöBEL, 'Every cotorsion-free algebra is an endomorphism algebra', Math. Z., 181 (1982), 451-470.

6. M. DUGAS AND R. GÖBEL, 'On endomorphism rings of primary abelian groups', Math. Ann., 261 (1982), 359-385.

7. M. DuGAS AND R. GöBEL, 'Countable mixed abelian groups with very nice full-rank subgroups', Israel J. Math., to appear.

8. M. Dugas, R. GöBel AND B. Goldsmith, 'Representation of algebras over a complete discrete valuation ring', Quart. J. Math. Oxford, to appear.

9. L. FucHs, Infinite abelian groups, Vol. I (Academic Press, New York, 1970); Vol. II (Academic Press, New York, 1973).

10. W. LIEBERT, 'Endomorphism rings of abelian p-groups', Studies on abelian groups (Paris, 1968), pp. 239-258. 
11. W. LIEBERT, 'Endomorphism rings of reduced torsion-free modules over complete discrete valuation rings', Trans. Amer. Math. Soc., 169 (1972), 347-363.

12. R. B. WARFIELD, 'The structure theory of mixed abelian groups', Abelian group theory, Lecture Notes in Mathematics 616 (eds. D. Arnold, R. Hunter and E. Walker, Berlin, 1977), pp. 1-38.

\author{
Department of Mathematics \\ Dublin Institute of Technology \\ Kevin Street \\ Dublin 8
}

Dublin Institute for Advanced Studies Dublin 4 